\title{
Iterated scaling limits for aggregation of random coefficient AR(1) and INAR(1) processes
}

\author{
FAnNi NedÉnyi ${ }^{* \diamond}$, Gyula PAP* \\ * Bolyai Institute, University of Szeged, Aradi vértanúk tere 1, H-6720 Szeged, \\ Hungary. \\ e-mails: nfanni@math.u-szeged.hu (F. Nedényi), papgy@math.u-szeged.hu (G. \\ Pap). \\ $\diamond$ Corresponding author.
}

$\|$

\begin{abstract}
We discuss joint temporal and contemporaneous aggregation of $N$ independent copies of strictly stationary $\mathrm{AR}(1)$ and INteger-valued AutoRegressive processes of order $1(\operatorname{INAR}(1))$ with random coefficient $\alpha \in(0,1)$ and idiosyncratic innovations. Assuming that $\alpha$ has a density function of the form $\psi(x)(1-x)^{\beta}, \quad x \in(0,1)$, with $\lim _{x \uparrow 1} \psi(x)=\psi_{1} \in(0, \infty)$, different Brownian limit processes of appropriately centered and scaled aggregated partial sums are shown to exist in case $\beta=1$ when taking first the limit as $N \rightarrow \infty$ and then the time scale $n \rightarrow \infty$, or vice versa. This paper completes the one of Pilipauskaite and Surgailis [4], and Barczy, Nedényi and Pap [1, where the iterated limits are given for every other possible value of the parameter $\beta$ for the two types of models.
\end{abstract}

\section{Introduction}

The aggregation problem is concerned with the relationship between individual (micro) behavior and aggregate (macro) statistics. There exist different types of aggregation. The scheme of contemporaneous (also called cross-sectional) aggregation of random-coefficient $\mathrm{AR}(1)$ models was firstly proposed by Robinson [8] and Granger [2] in order to obtain the long memory phenomena in aggregated time series.

Puplinskaitè and Surgailis [5, 6] discussed aggregation of random-coefficient $\mathrm{AR}(1)$ processes with infinite variance and innovations in the domain of attraction of a stable law. Related problems for some network traffic models, M/G/ $\infty$ queues with heavy-tailed activity periods, and renewal-reward processes have also been examined. On page 512 in Jirak [3] one can find many references for papers dealing

2010 Mathematics Subject Classifications: 60F05, 60J80, 60G15.

Key words and phrases: random coefficient $\operatorname{AR}(1)$ processes, random coefficient $\operatorname{INAR}(1)$ processes, temporal aggregation, contemporaneous aggregation, idiosyncratic innovations. 
with the aggregation of continuous time stochastic processes, and the introduction of Barczy, Nedényi and Pap [1] contains a detailed overview on the topic.

The aim of the present paper is to complete the papers of Pilipauskaite and Surgailis [4] and Barczy, Nedényi and Pap [1] by giving the appropriate iterated limit theorems for both the randomized $\mathrm{AR}(1)$ and INAR(1) models when the parameter $\beta=1$, which case is not investigated in both papers.

Let $\mathbb{Z}_{+}, \mathbb{N}, \mathbb{R}$ and $\mathbb{R}_{+}$denote the set of non-negative integers, positive integers, real numbers and non-negative real numbers, respectively. The paper of Pilipauskaitè and Surgailis [4] discusses the limit behavior of sums

$$
S_{t}^{(N, n)}:=\sum_{j=1}^{N} \sum_{k=1}^{\lfloor n t\rfloor} X_{k}^{(j)}, \quad t \in \mathbb{R}_{+}, \quad N, n \in \mathbb{N},
$$

where $\left(X_{k}^{(j)}\right)_{k \in \mathbb{Z}_{+}}, j \in \mathbb{N}$, are independent copies of a stationary random-coefficient $\mathrm{AR}(1)$ process

$$
X_{k}=\alpha X_{k-1}+\varepsilon_{k}, \quad k \in \mathbb{N}
$$

with standardized independent and identically distributed (i.i.d.) innovations $\left(\varepsilon_{k}\right)_{k \in \mathbb{N}}$ having $\mathbb{E}\left(\varepsilon_{1}\right)=0$ and $\operatorname{Var}\left(\varepsilon_{1}\right)=1$, and a random coefficient $\alpha$ with values in $[0,1)$, being independent of $\left(\varepsilon_{k}\right)_{k \in \mathbb{N}}$ and admitting a probability density function of the form

$$
\psi(x)(1-x)^{\beta}, \quad x \in[0,1)
$$

where $\beta \in(-1, \infty)$ and $\psi$ is an integrable function on $[0,1)$ having a limit $\lim _{x \uparrow 1} \psi(x)=\psi_{1}>0$. Here the distribution of $X_{0}$ is chosen as the unique stationary distribution of the model (1.2). Its existence was shown in Puplinskaitè and Surgailis [5, Proposition 1]. We point out that they considered so-called idiosyncratic innovations, i.e., the innovations $\left(\varepsilon_{k}^{(j)}\right)_{k \in \mathbb{N}}, j \in \mathbb{N}$, belonging to $\left(X_{k}^{(j)}\right)_{k \in \mathbb{Z}_{+}}$, $j \in \mathbb{N}$, are independent. In Pilipauskaitè and Surgailis [4] they derived scaling limits of the finite dimensional distributions of $\left(A_{N, n}^{-1} S_{t}^{(N, n)}\right)_{t \in \mathbb{R}_{+}}$, where $A_{N, n}$ are some scaling factors and first $N \rightarrow \infty$ and then $n \rightarrow \infty$, or vice versa, or both $N$ and $n$ increase to infinity, possibly with different rates. The iterated limit theorems for both orders of iteration are presented in the paper of Pilipauskaite and Surgailis [4, in Theorems 2.1 and 2.3, along with results concerning simultaneous limit theorems in Theorem 2.2 and 2.3. We note that the theorems cover different ranges of the possible values of $\beta \in(-1, \infty)$, namely, $\beta \in(-1,0), \beta=0, \beta \in(0,1)$, and $\beta>1$. Among the limit processes is a fractional Brownian motion, lines with random slopes where the slope is a stable variable, a stable Lévy process, and a Wiener process. Our paper deals with the missing case when $\beta=1$, for both two orders of iteration. 
The paper of Barczy, Nedényi and Pap [1] discusses the limit behavior of

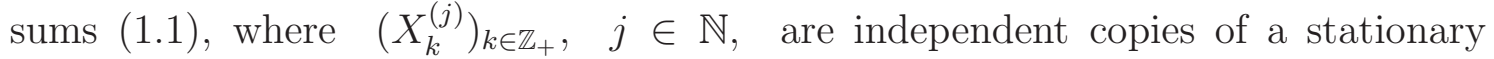
random-coefficient INAR(1) process. The usual INAR(1) process with non-randomcoefficient is defined as

$$
X_{k}=\sum_{j=1}^{X_{k-1}} \xi_{k, j}+\varepsilon_{k}, \quad k \in \mathbb{N}
$$

where $\left(\varepsilon_{k}\right)_{k \in \mathbb{N}}$ are i.i.d. non-negative integer-valued random variables, $\left(\xi_{k, j}\right)_{k, j \in \mathbb{N}}$ are i.i.d. Bernoulli random variables with mean $\alpha \in[0,1]$, and $X_{0}$ is a nonnegative integer-valued random variable such that $X_{0},\left(\xi_{k, j}\right)_{k, j \in \mathbb{N}}$ and $\left(\varepsilon_{k}\right)_{k \in \mathbb{N}}$ are independent. By using the binomial thinning operator $\alpha \circ$ due to Steutel and van Harn [9], the INAR(1) model in (1.4) can be considered as

$$
X_{k}=\alpha \circ X_{k-1}+\varepsilon_{k}, \quad k \in \mathbb{N},
$$

which form captures the resemblance with the $\mathrm{AR}(1)$ model. We note that an INAR(1) process can also be considered as a special branching process with immigration having Bernoulli offspring distribution.

We will consider a certain randomized INAR(1) process with randomized thinning parameter $\alpha$, given formally by the recursive equation (1.5), where $\alpha$ is a random variable with values in $(0,1)$. This means that, conditionally on $\alpha$, the process $\left(X_{k}\right)_{k \in \mathbb{Z}_{+}}$is an INAR(1) process with thinning parameter $\alpha$. Conditionally on $\alpha$, the i.i.d. innovations $\left(\varepsilon_{k}\right)_{k \in \mathbb{N}}$ are supposed to have a Poisson distribution with parameter $\lambda \in(0, \infty)$, and the conditional distribution of the initial value $X_{0}$ given $\alpha$ is supposed to be the unique stationary distribution, namely, a Poisson distribution with parameter $\lambda /(1-\alpha)$. For a rigorous construction of this process see Section 4 of Barczy, Nedényi and Pap [1]. The iterated limit theorems for both orders of iteration - that are analogous to the ones in case of the randomized $\mathrm{AR}(1)$ model - are presented in the latter paper, in Theorems 4.6-4.12. This paper deals with the missing case when $\beta=1$, for both two orders of iteration.

\section{Iterated aggregation of randomized INAR(1) processes with Poisson innovations}

Let $\alpha^{(j)}, j \in \mathbb{N}$, be a sequence of independent copies of the random variable $\alpha$, and let $\left(X_{k}^{(j)}\right)_{k \in \mathbb{Z}_{+}}, j \in \mathbb{N}$, be a sequence of independent copies of the process $\left(X_{k}\right)_{k \in \mathbb{Z}_{+}}$ with idiosyncratic innovations (i.e., the innovations $\left(\varepsilon_{k}^{(j)}\right)_{k \in \mathbb{N}}, j \in \mathbb{N}$, belonging to $\left(X_{k}^{(j)}\right)_{k \in \mathbb{Z}_{+}}, \quad j \in \mathbb{N}$, are independent) such that $\left(X_{k}^{(j)}\right)_{k \in \mathbb{Z}_{+}}$conditionally on $\alpha^{(j)}$ is a strictly stationary $\operatorname{INAR}(1)$ process with Poisson innovations for all $j \in \mathbb{N}$. 
First we examine a simple aggregation procedure. For each $N \in \mathbb{N}$, consider the stochastic process $\widetilde{S}^{(N)}=\left(\widetilde{S}_{k}^{(N)}\right)_{k \in \mathbb{Z}_{+}}$given by

$$
\widetilde{S}_{k}^{(N)}:=\sum_{j=1}^{N}\left(X_{k}^{(j)}-\mathbb{E}\left(X_{k}^{(j)} \mid \alpha^{(j)}\right)\right)=\sum_{j=1}^{N}\left(X_{k}^{(j)}-\frac{\lambda}{1-\alpha^{(j)}}\right), \quad k \in \mathbb{Z}_{+} .
$$

The following two propositions are Proposition 4.1 and 4.2 of Barczy, Nedényi and Pap [1]. We will use $\stackrel{\mathcal{D}_{\mathrm{f}}}{\longrightarrow}$ or $\mathcal{D}_{\mathrm{f}}$-lim for the weak convergence of the finite dimensional distributions.

2.1 Proposition. If $\mathbb{E}\left(\frac{1}{1-\alpha}\right)<\infty$, then

$$
N^{-\frac{1}{2}} \widetilde{S}^{(N)} \stackrel{\mathcal{D}_{\mathrm{f}}}{\longrightarrow} \widetilde{\mathcal{Y}} \quad \text { as } \quad N \rightarrow \infty
$$

where $\left(\widetilde{\mathcal{Y}}_{k}\right)_{k \in \mathbb{Z}_{+}}$is a stationary Gaussian process with zero mean and covariances

$$
\mathbb{E}\left(\widetilde{\mathcal{Y}}_{0} \widetilde{\mathcal{Y}}_{k}\right)=\operatorname{Cov}\left(X_{0}-\frac{\lambda}{1-\alpha}, X_{k}-\frac{\lambda}{1-\alpha}\right)=\lambda \mathbb{E}\left(\frac{\alpha^{k}}{1-\alpha}\right), \quad k \in \mathbb{Z}_{+} .
$$

2.2 Proposition. We have

$$
\left(n^{-\frac{1}{2}} \sum_{k=1}^{\lfloor n t\rfloor} \widetilde{S}_{k}^{(1)}\right)_{t \in \mathbb{R}_{+}}=\left(n^{-\frac{1}{2}} \sum_{k=1}^{\lfloor n t\rfloor}\left(X_{k}^{(1)}-\mathbb{E}\left(X_{k}^{(1)} \mid \alpha^{(1)}\right)\right)\right)_{t \in \mathbb{R}_{+}} \stackrel{\mathcal{D}_{\mathrm{f}}}{\longrightarrow} \frac{\sqrt{\lambda(1+\alpha)}}{1-\alpha} B
$$

as $n \rightarrow \infty$, where $B=\left(B_{t}\right)_{t \in \mathbb{R}_{+}}$is a standard Brownian motion, independent of $\alpha$.

In the forthcoming theorems we assume that the distribution of the random variable $\alpha$, i.e., the mixing distribution, has a probability density described in (1.3). We note that the form of this density function indicates $\beta>-1$. Furthermore, if $\alpha$ has such a density function, then for each $\ell \in \mathbb{N}$ the expectation $\mathbb{E}\left((1-\alpha)^{-\ell}\right)$ is finite if and only if $\beta>\ell-1$.

For each $N, n \in \mathbb{N}$, consider the stochastic process $\widetilde{S}^{(N, n)}=\left(\widetilde{S}_{t}^{(N, n)}\right)_{t \in \mathbb{R}_{+}}$given by

$$
\widetilde{S}_{t}^{(N, n)}:=\sum_{j=1}^{N} \sum_{k=1}^{\lfloor n t\rfloor}\left(X_{k}^{(j)}-\mathbb{E}\left(X_{k}^{(j)} \mid \alpha^{(j)}\right)\right), \quad t \in \mathbb{R}_{+}
$$

2.3 Theorem. If $\beta=1$, then

$$
\mathcal{D}_{\mathrm{f}}-\lim _{n \rightarrow \infty} \mathcal{D}_{\mathrm{f}}-\lim _{N \rightarrow \infty}(n \log n)^{-\frac{1}{2}} N^{-\frac{1}{2}} \widetilde{S}^{(N, n)}=\sqrt{2 \lambda \psi_{1}} B
$$

where $B=\left(B_{t}\right)_{t \in \mathbb{R}_{+}}$is a standard Wiener process. 
Proof of Theorem 2.3. Since $\mathbb{E}\left((1-\alpha)^{-1}\right)<\infty$, the condition in Proposition 2.1 is satisfied, meaning that

$$
N^{-\frac{1}{2}} \widetilde{S}^{(N)} \stackrel{\mathcal{D}_{\mathrm{f}}}{\longrightarrow} \widetilde{\mathcal{Y}} \quad \text { as } N \rightarrow \infty
$$

where $\left(\widetilde{\mathcal{Y}}_{k}\right)_{k \in \mathbb{Z}_{+}}$is a stationary Gaussian process with zero mean and covariances

$$
\mathbb{E}\left(\widetilde{\mathcal{Y}}_{0} \widetilde{\mathcal{Y}}_{k}\right)=\operatorname{Cov}\left(X_{0}-\frac{\lambda}{1-\alpha}, X_{k}-\frac{\lambda}{1-\alpha}\right)=\lambda \mathbb{E}\left(\frac{\alpha^{k}}{1-\alpha}\right), \quad k \in \mathbb{Z}_{+} .
$$

Therefore, it suffices to show that

$$
\mathcal{D}_{\mathrm{f}}-\lim _{n \rightarrow \infty} \frac{1}{\sqrt{n \log n}} \sum_{k=1}^{\lfloor n t\rfloor} \widetilde{\mathcal{Y}}_{k}=\sqrt{2 \lambda \psi_{1}} B
$$

where $B=\left(B_{t}\right)_{t \in \mathbb{R}_{+}}$is a standard Wiener process. This follows from the continuity theorem if for all $t_{1}, t_{2} \in \mathbb{N}$ we have

$$
\operatorname{Cov}\left(\frac{1}{\sqrt{n \log n}} \sum_{k=1}^{\left\lfloor n t_{1}\right\rfloor} \widetilde{\mathcal{Y}}_{k}, \frac{1}{\sqrt{n \log n}} \sum_{k=1}^{\left\lfloor n t_{2}\right\rfloor} \widetilde{\mathcal{Y}}_{k}\right) \rightarrow 2 \lambda \psi_{1} \min \left(t_{1}, t_{2}\right),
$$

as $n \rightarrow \infty$. By (2.1) we have

$$
\begin{aligned}
& \operatorname{Cov}\left(\frac{1}{\sqrt{n \log n}} \sum_{k=1}^{\left\lfloor n t_{1}\right\rfloor} \widetilde{\mathcal{Y}}_{k}, \frac{1}{\sqrt{n \log n}} \sum_{k=1}^{\left\lfloor n t_{2}\right\rfloor} \widetilde{\mathcal{Y}}_{k}\right)=\frac{\lambda}{n \log n} \mathbb{E}\left(\sum_{k=1}^{\left\lfloor n t_{1}\right\rfloor} \sum_{\ell=1}^{\left\lfloor n t_{2}\right\rfloor} \frac{\alpha^{|k-\ell|}}{1-\alpha}\right) \\
& =\frac{\lambda}{n \log n} \int_{0}^{1} \sum_{k=1}^{\left\lfloor n t_{1}\right\rfloor} \sum_{\ell=1}^{\left\lfloor n t_{2}\right\rfloor} \frac{a^{|k-\ell|}}{1-a} \psi(a)(1-a) \mathrm{d} a .
\end{aligned}
$$

First we derive

$$
\frac{1}{n \log n} \int_{0}^{1} \sum_{k=1}^{\left\lfloor n t_{1}\right\rfloor} \sum_{\ell=1}^{\left\lfloor n t_{2}\right\rfloor} a^{|k-\ell|} \mathrm{d} a \rightarrow 2 \min \left(t_{1}, t_{2}\right),
$$

as $n \rightarrow \infty$. Indeed, if we suppose that $t_{2}>t_{1}$, then

$$
\begin{aligned}
& \int_{0}^{1} \sum_{k=1}^{\left\lfloor n t_{1}\right\rfloor} \sum_{\ell=1}^{\left\lfloor n t_{2}\right\rfloor} a^{|k-\ell|} \mathrm{d} a=\sum_{k=1}^{\left\lfloor n t_{1}\right\rfloor} \sum_{\ell=1}^{\left\lfloor n t_{2}\right\rfloor} \frac{1}{|k-\ell|+1} \\
& =\left(\left\lfloor n t_{1}\right\rfloor+1\right)\left(H\left(\left\lfloor n t_{1}\right\rfloor\right)-1\right)+2-\left\lfloor n t_{1}\right\rfloor+\left\lfloor n t_{1}\right\rfloor\left(H\left(\left\lfloor n t_{2}\right\rfloor\right)-1\right) \\
& \quad+\left(\left\lfloor n t_{2}\right\rfloor-\left\lfloor n t_{1}\right\rfloor+1\right)\left(H\left(\left\lfloor n t_{2}\right\rfloor\right)-H\left(\left\lfloor n t_{2}\right\rfloor-\left\lfloor n t_{1}\right\rfloor+1\right)\right) \\
& =\left(\left\lfloor n t_{1}\right\rfloor+1\right)\left(\log \left(\left\lfloor n t_{1}\right\rfloor\right)+O(1)\right)+2-\left\lfloor n t_{1}\right\rfloor+\left\lfloor n t_{1}\right\rfloor\left(\log \left\lfloor n t_{2}\right\rfloor+O(1)\right) \\
& \quad+\left(\left\lfloor n t_{2}\right\rfloor-\left\lfloor n t_{1}\right\rfloor+1\right)\left(\log \left(\left\lfloor n t_{2}\right\rfloor\right)-\log \left(\left\lfloor n t_{2}\right\rfloor-\left\lfloor n t_{1}\right\rfloor+1\right)+O(1)\right),
\end{aligned}
$$


where $H(n)$ denotes the $n$-th harmonic number, and it is well known that $H(n)=$ $\log n+O(1)$ for every $n \in \mathbb{N}$. Therefore, convergence (2.3) holds. Consequently, (2.2) will follow from

$$
I_{n}:=\frac{1}{n \log n} \int_{0}^{1} \sum_{k=1}^{\left\lfloor n t_{1}\right\rfloor} \sum_{\ell=1}^{\left\lfloor n t_{2}\right\rfloor} a^{|k-\ell|}\left|\psi(a)-\psi_{1}\right| \mathrm{d} a \rightarrow 0
$$

as $n \rightarrow \infty$. Note that for every $\varepsilon>0$ there is a $\delta_{\varepsilon}>0$ such that for every $a \in\left(1-\delta_{\varepsilon}, 1\right)$ it holds that $\left|\psi(a)-\psi_{1}\right|<\varepsilon$. Hence

$$
\begin{aligned}
n \log n I_{n} & \leqslant \int_{0}^{1-\delta_{\varepsilon}} \sum_{k=1}^{\left\lfloor n t_{1}\right\rfloor} \sum_{\ell=1}^{\left\lfloor n t_{2}\right\rfloor} a^{|k-\ell|}\left(\psi(a)+\psi_{1}\right) \mathrm{d} a+\int_{1-\delta_{\varepsilon}}^{1} \sum_{k=1}^{\left\lfloor n t_{1}\right\rfloor} \sum_{\ell=1}^{\left\lfloor n t_{2}\right\rfloor} a^{|k-\ell|}\left|\psi(a)-\psi_{1}\right| \mathrm{d} a \\
& \leqslant \int_{0}^{1-\delta_{\varepsilon}} \frac{2\left\lfloor n t_{1}\right\rfloor}{\delta_{\varepsilon}}\left(\psi(a)+\psi_{1}\right) \mathrm{d} a+\varepsilon \int_{1-\delta_{\varepsilon}}^{1} \sum_{k=1}^{\left\lfloor n t_{1}\right\rfloor} \sum_{\ell=1}^{\left\lfloor n t_{2}\right\rfloor} a^{|k-\ell|} \mathrm{d} a,
\end{aligned}
$$

meaning that for every $\varepsilon>0$ by (2.3) we have $\limsup _{n \rightarrow \infty}\left|I_{n}\right| \leqslant 0+$ $\varepsilon 4 \psi_{1} \min \left(t_{1}, t_{2}\right)$, resulting that $\lim _{n \rightarrow \infty} I_{n}=0$, which completes the proof.

2.4 Theorem. If $\beta=1$, then

$$
\mathcal{D}_{\mathrm{f}}-\lim _{N \rightarrow \infty} \mathcal{D}_{\mathrm{f}}-\lim _{n \rightarrow \infty} \frac{1}{\sqrt{n N \log N}} \widetilde{S}^{(N, n)}=\sqrt{\lambda \psi_{1}} B,
$$

where $B=\left(B_{t}\right)_{t \in \mathbb{R}_{+}}$is a standard Wiener process.

Proof of Theorem 2.4. By the second proof of Theorem 4.9 of Barczy, Nedényi and Pap [1] it suffices to show that

$$
\frac{1}{N \log N} \sum_{j=1}^{N} \frac{\lambda\left(1+\alpha^{(j)}\right)}{\left(1-\alpha^{(j)}\right)^{2}} \stackrel{\mathcal{D}}{\longrightarrow} \lambda \psi_{1}, \quad N \rightarrow \infty .
$$

Let us apply Theorem 7.1 of Resnick [7] with

$$
X_{N, j}:=\frac{1}{N} \frac{\lambda\left(1+\alpha^{(j)}\right)}{\left(1-\alpha^{(j)}\right)^{2}}
$$

meaning that

$$
N \mathbb{P}\left(X_{N, 1}>x\right)=N \mathbb{P}\left(\frac{\lambda(1+\alpha)}{(1-\alpha)^{2}}>N x\right)=N \int_{1-\widetilde{h}(\lambda, N x)}^{1} \psi(a)(1-a) \mathrm{d} a,
$$

where $\tilde{h}(\lambda, x)=(1 / 4+\sqrt{1 / 16+x /(2 \lambda)})^{-1}$. Note that for every $\varepsilon>0$ there is a $\delta_{\varepsilon}>0$ such that for every $a \in\left(1-\delta_{\varepsilon}, 1\right)$ it holds that $\left|\psi(a)-\psi_{1}\right|<\varepsilon$. Then,

$$
N \int_{1-\widetilde{h}(\lambda, N x)}^{1}\left|\psi(a)-\psi_{1}\right|(1-a) \mathrm{d} a \leqslant N \varepsilon \frac{(\widetilde{h}(\lambda, N x))^{2}}{2} \leqslant \frac{\varepsilon \lambda}{x}
$$


for every $x>0$ and large enough $N$. Therefore, for every $x>0$ we have

$$
\begin{aligned}
& \lim _{N \rightarrow \infty} N \mathbb{P}\left(X_{N, 1}>x\right)=\lim _{N \rightarrow \infty} N \int_{1-\widetilde{h}(\lambda, N x)}^{1} \psi_{1}(1-a) \mathrm{d} a \\
& =\lim _{N \rightarrow \infty} N \psi_{1} \frac{(\widetilde{h}(\lambda, N x))^{2}}{2}=\lim _{N \rightarrow \infty} \frac{\psi_{1}}{2} \frac{N}{\left(\frac{1}{4}+\sqrt{\frac{1}{16}+\frac{N x}{2 \lambda}}\right)^{2}}=\frac{\psi_{1} \lambda}{x}=: \nu([x, \infty)),
\end{aligned}
$$

where $\nu$ is obviously a Lévy-measure. By the decomposition

$$
N \mathbb{E}\left(X_{N, 1}^{2} \mathbb{1}_{\left\{\left|X_{N, 1}\right| \leqslant \varepsilon\right\}}\right)=N \int_{0}^{1-\widetilde{h}(\lambda, N \varepsilon)}\left(\frac{\lambda(1+a)}{N(1-a)^{2}}\right)^{2} \psi(a)(1-a) \mathrm{d} a=I_{N}^{(1)}+I_{N}^{(2)},
$$

where

$$
I_{N}^{(1)}:=N \int_{0}^{1-\delta_{\varepsilon}}\left(\frac{\lambda(1+a)}{N(1-a)^{2}}\right)^{2} \psi(a)(1-a) \mathrm{d} a \leqslant \frac{1}{N} \lambda^{2} \frac{2^{2}}{\delta_{\varepsilon}^{4}} 1 \rightarrow 0
$$

as $N \rightarrow \infty$, and

$$
\begin{aligned}
I_{N}^{(2)} & :=N \int_{1-\delta_{\varepsilon}}^{1-\widetilde{h}(\lambda, N \varepsilon)}\left(\frac{\lambda(1+a)}{N(1-a)^{2}}\right)^{2} \psi(a)(1-a) \mathrm{d} a \\
& \leqslant \frac{8 \psi_{1} \lambda^{2}}{N} \int_{1-\delta_{\varepsilon}}^{1-\widetilde{h}(\lambda, N \varepsilon)} \frac{\mathrm{d} a}{(1-a)^{3}}=\frac{4 \psi_{1} \lambda^{2}}{N}\left[\widetilde{h}(\lambda, N \varepsilon)^{-2}-\delta_{\varepsilon}^{-2}\right] \leqslant 8 \psi_{1} \lambda^{2} \varepsilon
\end{aligned}
$$

for large enough $N$ values, so it follows that

$$
\lim _{\varepsilon \rightarrow 0} \limsup _{N \rightarrow \infty} N \mathbb{E}\left(X_{N, 1}^{2} \mathbb{1}_{\left\{\left|X_{N, 1}\right| \leqslant \varepsilon\right\}}\right)=0 .
$$

Therefore, by applying Theorem 7.1 of Resnick [7] with the choice $t=1$ we get that

$$
\begin{aligned}
& \sum_{j=1}^{N}\left[\frac{\lambda\left(1+\alpha^{(j)}\right)}{N\left(1-\alpha^{(j)}\right)^{2}}-\mathbb{E}\left(\frac{\lambda(1+\alpha)}{N(1-\alpha)^{2}} \mathbb{1}_{\left\{\frac{\lambda(1+\alpha)}{N(1-\alpha)^{2}} \leqslant 1\right\}}\right)\right] \\
& =\sum_{j=1}^{N}\left[\frac{\lambda\left(1+\alpha^{(j)}\right)}{N\left(1-\alpha^{(j)}\right)^{2}}-\frac{\lambda \psi_{1}}{N} \int_{0}^{1-\sqrt{\frac{2 \lambda}{N}}} \frac{2}{(1-a)^{2}}(1-a) \mathrm{d} a\right. \\
& +\frac{\lambda \psi_{1}}{N} \int_{0}^{1-\sqrt{\frac{2 \lambda}{N}}} \frac{2}{(1-a)^{2}}(1-a) \mathrm{d} a-\frac{\lambda \psi_{1}}{N} \int_{0}^{1-\widetilde{h}(\lambda, N)} \frac{2}{(1-a)^{2}}(1-a) \mathrm{d} a \\
& +\frac{\lambda \psi_{1}}{N} \int_{0}^{1-\widetilde{h}(\lambda, N)} \frac{2}{(1-a)^{2}}(1-a) \mathrm{d} a-\frac{\lambda \psi_{1}}{N} \int_{0}^{1-\widetilde{h}(\lambda, N)} \frac{1+a}{(1-a)^{2}}(1-a) \mathrm{d} a \\
& \left.\quad+\frac{\lambda \psi_{1}}{N} \int_{0}^{1-\widetilde{h}(\lambda, N)} \frac{1+a}{(1-a)^{2}}(1-a) \mathrm{d} a-\frac{\lambda}{N} \int_{0}^{1-\widetilde{h}(\lambda, N)} \frac{1+a}{(1-a)^{2}} \psi(a)(1-a) \mathrm{d} a\right] \\
& =
\end{aligned}
$$


where by (5.37) of Resnick [7]

$$
\mathbb{E}\left(\mathrm{e}^{\mathrm{i} \theta X_{0}}\right)=\exp \left\{\int_{1}^{\infty}\left(\mathrm{e}^{\mathrm{i} \theta x}-1\right) \frac{\psi_{1} \lambda \mathrm{d} x}{x^{2}}+\int_{0}^{1}\left(\mathrm{e}^{\mathrm{i} \theta x}-1-\mathrm{i} \theta x\right) \frac{\psi_{1} \lambda \mathrm{d} x}{x^{2}}\right\}, \quad \theta \in \mathbb{R} .
$$

We show that

$$
\frac{\left|J_{N}^{(1)}\right|+\left|J_{N}^{(2)}\right|+\left|J_{N}^{(3)}\right|}{\log N} \rightarrow 0, \quad N \rightarrow \infty
$$

resulting

$$
\begin{gathered}
\frac{1}{\log N} \sum_{j=1}^{N} \frac{\lambda\left(1+\alpha^{(j)}\right)}{N\left(1-\alpha^{(j)}\right)^{2}}=\frac{1}{\log N} \sum_{j=1}^{N}\left[\frac{\lambda\left(1+\alpha^{(j)}\right)}{N\left(1-\alpha^{(j)}\right)^{2}}-\frac{\lambda \psi_{1}}{N} \int_{0}^{1-\sqrt{\frac{2 \lambda}{N}}} \frac{2}{1-a} \mathrm{~d} a\right] \\
\quad+\frac{2 \lambda \psi_{1}}{\log N}\left(-\log \left(\sqrt{\frac{2 \lambda}{N}}\right)\right) \stackrel{\mathcal{D}}{\longrightarrow} 0 \cdot X_{0}+\lambda \psi_{1}=\lambda \psi_{1}, \quad N \rightarrow \infty .
\end{gathered}
$$

Indeed,

$$
\frac{J_{N}^{(1)}}{\log N}=\frac{\psi_{1}}{\log N} \int_{1-\sqrt{\frac{2 \lambda}{N}}}^{1-\widetilde{h}(\lambda, N)} \frac{2}{1-a} \mathrm{~d} a=\frac{2 \psi_{1}}{\log N} \log \left(\sqrt{\frac{2 \lambda}{N}}\left(\frac{1}{4}+\sqrt{\frac{1}{16}+\frac{N}{2 \lambda}}\right)\right)
$$

converges to 0 as $N \rightarrow \infty$. Moreover,

$$
\frac{J_{N}^{(2)}}{\log N}=\frac{\psi_{1}}{\log N} \int_{0}^{1-\widetilde{h}(\lambda, N)} \frac{1-a}{(1-a)^{2}}(1-a) \mathrm{d} a=\frac{\psi_{1}}{\log N}\left(1-\frac{1}{\frac{1}{4}+\sqrt{\frac{1}{16}+\frac{N}{2 \lambda}}}\right)
$$

converges to 0 as $N \rightarrow \infty$. Finally,

$$
\begin{aligned}
& \left|\frac{J_{N}^{(3)}}{\log N}\right|=\left|\frac{1}{\log N} \int_{0}^{1-\widetilde{h}(\lambda, N)} \frac{1+a}{1-a}\left(\psi_{1}-\psi(a)\right) \mathrm{d} a\right| \\
& \leqslant \frac{1}{\log N} \int_{0}^{1-\delta_{\varepsilon}} \frac{2}{\delta_{\varepsilon}}\left(\psi_{1}+\psi(a)\right) \mathrm{d} a+\frac{1}{\log N} \int_{1-\delta_{\varepsilon}}^{1-\widetilde{h}(\lambda, N)} \frac{2}{1-a} \varepsilon \mathrm{d} a \\
& \leqslant \frac{1}{\log N} \frac{2}{\delta_{\varepsilon}}\left(\psi_{1}+\delta_{\varepsilon}^{-1}\right)+\frac{2 \varepsilon}{\log N}\left[\log \delta_{\varepsilon}+\log \left(\frac{1}{4}+\sqrt{\frac{1}{16}+\frac{N}{2 \lambda}}\right) .\right],
\end{aligned}
$$

One can easily see that for all $\varepsilon>0$, we get $\lim _{\sup _{N \rightarrow \infty}}\left|J_{N}^{(3)} / \log N\right| \leqslant 0+\varepsilon$, resulting that $\lim _{N \rightarrow \infty} J_{N}^{(3)} / \log N=0$, which completes the proof.

\section{Iterated aggregation of randomized AR(1) pro- cesses with Gaussian innovations}

Let $\alpha^{(j)}, j \in \mathbb{N}$, be a sequence of independent copies of the random variable $\alpha$, and let $\left(X_{k}^{(j)}\right)_{k \in \mathbb{Z}_{+}}, \quad j \in \mathbb{N}$, be a sequence of independent copies of the process 
$\left(X_{k}\right)_{k \in \mathbb{Z}_{+}}$with idiosyncratic Gaussian innovations (i.e., the innovations $\left(\varepsilon_{k}^{(j)}\right)_{k \in \mathbb{Z}_{+}}$, $j \in \mathbb{N}$, belonging to $\left(X_{k}^{(j)}\right)_{k \in \mathbb{Z}_{+}}, \quad j \in \mathbb{N}$, are independent) having zero mean and variance $\sigma^{2} \in \mathbb{R}_{+}$such that $\left(X_{k}^{(j)}\right)_{k \in \mathbb{Z}_{+}}$conditionally on $\alpha^{(j)}$ is a strictly stationary $\mathrm{AR}(1)$ process for all $j \in \mathbb{N}$. A rigorous construction of this randomcoefficient process can be given similarly as in case of the randomized INAR(1) process detailed in Section 4 of Barczy, Nedényi and Pap [1].

First we examine a simple aggregation procedure. For each $N \in \mathbb{N}$, consider the stochastic process $\widetilde{S}^{(N)}=\left(\widetilde{S}_{k}^{(N)}\right)_{k \in \mathbb{Z}_{+}}$given by

$$
\widetilde{S}_{k}^{(N)}:=\sum_{j=1}^{N} X_{k}^{(j)}, \quad k \in \mathbb{Z}_{+}
$$

The following two propositions are the counterparts of Proposition 2.1] and 2.2. and can be proven similarly as the two concerning the randomized $\operatorname{INAR}(1)$ process.

3.1 Proposition. If $\mathbb{E}\left(\frac{1}{1-\alpha^{2}}\right)<\infty$, then

$$
N^{-\frac{1}{2}} \widetilde{S}^{(N)} \stackrel{\mathcal{D}_{\mathrm{f}}}{\longrightarrow} \widetilde{\mathcal{Y}} \quad \text { as } N \rightarrow \infty
$$

where $\left(\widetilde{\mathcal{Y}}_{k}\right)_{k \in \mathbb{Z}_{+}}$is a stationary Gaussian process with zero mean and covariances

$$
\mathbb{E}\left(\widetilde{\mathcal{Y}}_{0} \widetilde{\mathcal{Y}}_{k}\right)=\operatorname{Cov}\left(X_{0}, X_{k}\right)=\sigma^{2} \mathbb{E}\left(\frac{\alpha^{k}}{1-\alpha^{2}}\right), \quad k \in \mathbb{Z}_{+}
$$

3.2 Proposition. We have

$$
\left(n^{-\frac{1}{2}} \sum_{k=1}^{\lfloor n t\rfloor} \widetilde{S}_{k}^{(1)}\right)_{t \in \mathbb{R}_{+}}=\left(n^{-\frac{1}{2}} \sum_{k=1}^{\lfloor n t\rfloor} X_{k}^{(1)}\right)_{t \in \mathbb{R}_{+}} \stackrel{\mathcal{D}_{\mathrm{f}}}{\longrightarrow} \frac{\sigma}{1-\alpha} B
$$

as $n \rightarrow \infty$, where $B=\left(B_{t}\right)_{t \in \mathbb{R}_{+}}$is a standard Brownian motion, independent of $\alpha$.

Again, we assume that the distribution of the random variable $\alpha$ has a probability density described in (1.3). Note that for each $\ell \in \mathbb{N}$ the expectation $\mathbb{E}\left(\left(1-\alpha^{2}\right)^{-\ell}\right)$ is finite if and only if $\beta>\ell-1$.

For each $N, n \in \mathbb{N}$, consider the stochastic process $\widetilde{S}^{(N, n)}=\left(\widetilde{S}_{t}^{(N, n)}\right)_{t \in \mathbb{R}_{+}}$given by

$$
\widetilde{S}_{t}^{(N, n)}:=\sum_{j=1}^{N} \sum_{k=1}^{\lfloor n t\rfloor} X_{k}^{(j)}, \quad t \in \mathbb{R}_{+} .
$$

3.3 Theorem. If $\beta=1$, then

$$
\mathcal{D}_{\mathrm{f}}-\lim _{n \rightarrow \infty} \mathcal{D}_{\mathrm{f}}-\lim _{N \rightarrow \infty}(n \log n)^{-\frac{1}{2}} N^{-\frac{1}{2}} \widetilde{S}^{(N, n)}=\sqrt{\sigma^{2} \psi_{1}} B,
$$

where $B=\left(B_{t}\right)_{t \in \mathbb{R}_{+}}$is a standard Wiener process. 
Proof of Theorem 3.3. Since $\mathbb{E}\left(\left(1-\alpha^{2}\right)^{-1}\right)<\infty$, the condition in Proposition 3.1 is satisfied, meaning that

$$
N^{-\frac{1}{2}} \widetilde{S}^{(N)} \stackrel{\mathcal{D}_{\mathrm{f}}}{\longrightarrow} \widetilde{\mathcal{Y}} \quad \text { as } N \rightarrow \infty
$$

where $\left(\widetilde{\mathcal{Y}}_{k}\right)_{k \in \mathbb{Z}_{+}}$is a stationary Gaussian process with zero mean and covariances

$$
\mathbb{E}\left(\widetilde{\mathcal{Y}}_{0} \widetilde{\mathcal{Y}}_{k}\right)=\operatorname{Cov}\left(X_{0}, X_{k}\right)=\sigma^{2} \mathbb{E}\left(\frac{\alpha^{k}}{1-\alpha^{2}}\right), \quad k \in \mathbb{Z}_{+}
$$

Therefore, it suffices to show that

$$
\mathcal{D}_{\mathrm{f}^{-}} \lim _{n \rightarrow \infty} \frac{1}{\sqrt{n \log n}} \sum_{k=1}^{\lfloor n t\rfloor} \widetilde{\mathcal{Y}}_{k}=\sqrt{\sigma^{2} \psi_{1}} B
$$

where $B=\left(B_{t}\right)_{t \in \mathbb{R}_{+}}$is a standard Wiener process. This follows from the continuity theorem, if for all $t_{1}, t_{2} \in \mathbb{N}$ we have

$$
\operatorname{Cov}\left(\frac{1}{\sqrt{n \log n}} \sum_{k=1}^{\left\lfloor n t_{1}\right\rfloor} \widetilde{\mathcal{Y}}_{k}, \frac{1}{\sqrt{n \log n}} \sum_{k=1}^{\left\lfloor n t_{2}\right\rfloor} \widetilde{\mathcal{Y}}_{k}\right) \rightarrow \sigma^{2} \psi_{1} \min \left(t_{1}, t_{2}\right), \quad n \rightarrow \infty
$$

It is known that

$$
\begin{aligned}
& \operatorname{Cov}\left(\frac{1}{\sqrt{n \log n}} \sum_{k=1}^{\left\lfloor n t_{1}\right\rfloor} \widetilde{\mathcal{Y}}_{k}, \frac{1}{\sqrt{n \log n}} \sum_{k=1}^{\left\lfloor n t_{2}\right\rfloor} \widetilde{\mathcal{Y}}_{k}\right)=\frac{\sigma^{2}}{n \log n} \mathbb{E}\left(\sum_{k=1}^{\left\lfloor n t_{1}\right\rfloor} \sum_{\ell=1}^{\left\lfloor n t_{2}\right\rfloor} \frac{\alpha^{|k-\ell|}}{1-\alpha^{2}}\right) \\
& =\frac{\sigma^{2}}{n \log n} \int_{0}^{1} \sum_{k=1}^{\left\lfloor n t_{1}\right\rfloor} \sum_{\ell=1}^{\left\lfloor n t_{2}\right\rfloor} \frac{a^{\lfloor k-\ell \mid}}{1-a^{2}} \psi(a)(1-a) \mathrm{d} a \\
& =\frac{\sigma^{2}}{n \log n} \int_{0}^{1} \sum_{k=1}^{\left\lfloor n t_{1}\right\rfloor} \sum_{\ell=1}^{\left\lfloor n t_{2}\right\rfloor} a^{|k-\ell|} \psi(a) \mathrm{d} a-\frac{\sigma^{2}}{n \log n} \int_{0}^{1} \sum_{k=1}^{\left\lfloor n t_{1}\right\rfloor} \sum_{\ell=1}^{\left\lfloor n t_{2}\right\rfloor} \frac{a^{|k-\ell|+1}}{1+a} \psi(a) \mathrm{d} a
\end{aligned}
$$

It was shown in the proof of Theorem 2.3 that

$$
\frac{\sigma^{2}}{n \log n} \int_{0}^{1} \sum_{k=1}^{\left\lfloor n t_{1}\right\rfloor} \sum_{\ell=1}^{\left\lfloor n t_{2}\right\rfloor} a^{|k-\ell|} \psi(a) \mathrm{d} a \rightarrow 2 \sigma^{2} \psi_{1} \min \left(t_{1}, t_{2}\right), \quad n \rightarrow \infty .
$$

We are going to prove that

$$
\frac{\sigma^{2}}{n \log n} \int_{0}^{1} \sum_{k=1}^{\left\lfloor n t_{1}\right\rfloor} \sum_{\ell=1}^{\left\lfloor n t_{2}\right\rfloor} \frac{a^{|k-\ell|+1}}{1+a} \psi(a) \mathrm{d} a-\frac{\sigma^{2}}{n \log n} \int_{0}^{1} \sum_{k=1}^{\left\lfloor n t_{1}\right\rfloor} \sum_{\ell=1}^{\left\lfloor n t_{2}\right\rfloor} \frac{a^{|k-\ell|}}{1+a} \psi(a) \mathrm{d} a
$$

converges to 0 as $n \rightarrow \infty$, which proves our theorem. Indeed, if $t_{2}>t_{1}$, then

$$
\begin{aligned}
& \left|\sum_{k=1}^{\left\lfloor n t_{1}\right\rfloor} \sum_{\ell=1}^{\left\lfloor n t_{2}\right\rfloor}\left(\frac{a^{\lfloor k-\ell \mid+1}}{1+a}-\frac{a^{|k-\ell|}}{1+a}\right)\right|=\frac{1}{1+a}\left|\sum_{k=1}^{\left\lfloor n t_{1}\right\rfloor}\left(a^{k}-(a+1)+a^{\left\lfloor n t_{2}\right\rfloor-k+1}\right)\right| \\
& =\frac{1}{1+a}\left|\frac{a\left(a^{\left\lfloor n t_{1}\right\rfloor}-1\right)}{a-1}-(a+1)\left\lfloor n t_{1}\right\rfloor+\frac{a^{\left\lfloor n t_{2}\right\rfloor+1}-a^{\left\lfloor n t_{2}\right\rfloor-\left\lfloor n t_{1}\right\rfloor+1}}{a-1}\right| \leqslant 4\left\lfloor n t_{2}\right\rfloor,
\end{aligned}
$$


and as $\psi(a), a \in(0,1)$ is integrable,

$$
\frac{\sigma^{2}}{n \log n} \int_{0}^{1} 4\left\lfloor n t_{2}\right\rfloor \psi(a) \mathrm{d} a \rightarrow 0, \quad n \rightarrow \infty .
$$

This completes the proof.

3.4 Theorem. If $\beta=1$, then

$$
\mathcal{D}_{\mathrm{f}}-\lim _{N \rightarrow \infty} \mathcal{D}_{\mathrm{f}}-\lim _{n \rightarrow \infty} \frac{1}{\sqrt{n N \log N}} \widetilde{S}^{(N, n)}=\sqrt{\frac{\sigma^{2} \psi_{1}}{2}} B,
$$

where $B=\left(B_{t}\right)_{t \in \mathbb{R}_{+}}$is a standard Wiener process.

The proof is similar to the INAR(1) case since the only difference is a missing $1+\alpha$ factor in the numerator and the constants.

\section{References}

[1] M. Barczy, F. Nedényi, and G. Pap. Iterated scaling limits for aggregation of randomized INAR(1) processes with idiosyncratic Poisson innovations. arXiv:1509.05149, 2015.

[2] C. W. J. Granger. Long memory relationships and the aggregation of dynamic models. J. Econometrics, 14(2):227-238, 1980.

[3] M. Jirak. Limit theorems for aggregated linear processes. Adv. in Appl. Probab., $45(2): 520-544,2013$.

[4] V. Pilipauskaité and D. Surgailis. Joint temporal and contemporaneous aggregation of random-coefficient $\mathrm{AR}(1)$ processes. Stochastic Process. Appl., 124(2):1011-1035, 2014.

[5] D. Puplinskaitė and D. Surgailis. Aggregation of random-coefficient AR(1) process with infinite variance and common innovations. Lith. Math. J., 49(4):446463, 2009.

[6] D. Puplinskaitè and D. Surgailis. Aggregation of a random-coefficient AR(1) process with infinite variance and idiosyncratic innovations. Adv. in Appl. Probab., 42(2):509-527, 2010.

[7] S. I. Resnick. Heavy-Tail Phenomena. Springer, New York, 2007.

[8] P. M. Robinson. Statistical inference for a random coefficient autoregressive model. Scand. J. Statist., 5(3):163-168, 1978.

[9] F. W. Steutel and K. van Harn. Discrete analogues of self-decomposability and stability. Ann. Probab., 7(5):893-899, 1979. 